\title{
Low-energy astrophysics with KamLAND
}

\section{Nanami Kawada, ${ }^{a, *}$ Shuhei Obara $^{b}$ and Koji Ishidoshiro ${ }^{a}$ on behalf of the KamLAND Collaboration}

(a complete list of authors can be found at the end of the proceedings)

${ }^{a}$ Research Center for Neutrino Science, Tohoku University, Sendai 980-8578, Japan

${ }^{b}$ Frontier Research Institute for Interdisciplinary Sciences, Tohoku University, Sendai 980-8578, Japan

E-mail: kawada@awa.tohoku.ac.jp

We present two results of a search for $\mathrm{MeV}$-scale neutrino and anti-neutrino events correlated with gravitational wave events/candidates and large solar flares with KamLAND.

The KamLAND detector is a large-volume neutrino detector using liquid scintillator, which is located at $1 \mathrm{~km}$ underground under the top of Mt. Ikenoyama in Kamioka, Japan. KamLAND has multiple reaction channels to detect neutrinos. Electron antineutrino can be detected via inversebeta decay with $1.8 \mathrm{MeV}$ neutrino energy threshold. All flavors of neutrinos can be detected via neutrino-electron scattering without neutrino energy threshold. KamLAND has continued the neutrino observation since 2002 March.

We use the data set of 60 gravitational waves provided by the LIGO/Virgo collaboration during their second and third observing runs and search for coincident electron antineutrino events in KamLAND. We find no significant coincident signals within a $\pm 500 \mathrm{~s}$ timing window from each gravitational wave and present $90 \%$ C.L. upper limits on the electron antineutrino fluence between $10^{8}-10^{13} \mathrm{~cm}^{-2}$ for neutrino energies of $1.8-111 \mathrm{MeV}$.

For a solar-flare neutrino search at KamLAND, we determine the timing window using the solar X-ray data set provided by the GOES satellite series from 2002 to 2019 and search for the excess of coincident event rate on the all-flavor neutrinos. We find no significant event rate excess in the flare time windows and get 90\% C.L. upper limits on the fluence of neutrinos of all flavors (electron anti-neutrinos) between $10^{10}-10^{13} \mathrm{~cm}^{-2}\left(10^{8}-10^{13} \mathrm{~cm}^{-2}\right)$ for neutrino energies in the energy range of $0.4-35 \mathrm{MeV}$.

$37^{\text {th }}$ International Cosmic Ray Conference (ICRC 2021)

July 12th - 23rd, 2021

Online-Berlin, Germany

\footnotetext{
*Presenter
} 


\section{The KamLAND experiment}

\subsection{KamLAND detector}

The KamLAND detector is the largest liquid-scintillator-based anti-neutrino detector in operation since 2002, which is located about $1 \mathrm{~km}$ underground ( $2700 \mathrm{~m}$ water equivalent) under Mt. Ikenoyama in Kamioka, Japan. Rock above the detector reduces the muon flux produced as secondary particle of cosmic rays by five orders of magnitude.

KamLAND consists of a $10 \mathrm{~m}$-radius $\times 20 \mathrm{~m}$-height cylindrical tank (outer detector) and a $9 \mathrm{~m}$ radius spherical stainless steel tank (inner detector). Neutrinos are detected by the inner detector (ID). The main volume of ID is $1 \mathrm{kt}$ liquid scintillator and supported by a $6.5 \mathrm{~m}$-radius nylon/EVOH balloon installed at the center of the ID. This nylon/EVOH balloon is called the outer balloon. The scintillation photons are counted by 1325 17-inch Photo Multiplier Tubes (PMTs) and 554 20-inch PMTs mounted on the inner surface of the spherical tank. Non-scintillating mineral oil is filled between the outer balloon and the spherical tank to suppress backgrounds from radioactive impurities in PMTs. The outer detector (OD) is a water-cherenkov detector using $3.2 \mathrm{kt}$ pure water for shielding from external $\gamma$-ray backgrounds. An active muon counter is provided by 25520 -inch PMTs before a refurbishment in 2016 and 140 20-inch PMTs after the refurbishment [1]. The details of the KamLAND detector are summarized in [2].

Since August 2011, KamLAND has searched for the neutrinoless double-beta decay of ${ }^{136} \mathrm{Xe}$ using a nylon balloon (inner balloon) installed at the center of the detector, which is filled with xenon-loaded liquid scintillator [3]. The inner balloon radius was $1.5 \mathrm{~m}$ and the mass of xenon was about $400 \mathrm{~kg}$ in the first phase, which ran from August 2011 to September 2015, called KamLANDZen 400. In 2018 May, the KamLAND-Zen experiment was updated to KamLAND-Zen 800 using 1.9 m-radius inner balloon and the double amount of xenon for a higher sensitivity [4]. In these KamLAND-Zen periods, the region with xenon-loaded liquid scintillator was excluded from the target volume for the neutrino observation to suppress backgrounds from xenon nuclei, the inner balloon and supporting structure.

\subsection{Neutrino detection in KamLAND}

KamLAND has multiple neutrino-detection channels. We used the following two reaction channels; inverse-beta decay(IBD), $\bar{v}_{e}+p \rightarrow e^{+}+n$, and neutrino-electron scattering(ES), $v+e^{-} \rightarrow$ $v+e^{-}$in this study.

IBD is sensitive only to electron anti-neutrino $\left(\bar{v}_{e}\right)$ with energy more than $1.8 \mathrm{MeV}$. But it is a strong advantage of this channel that the cross section is roughly 10 times larger than that of ES. In addition, this channel can suppress the background by a delayed-coincidence method. The positron's kinetic energy and the two $\gamma$-rays from annihilation with electron are observed as one event called prompt event. The incident neutrino energy, $E_{v}$, can be reconstructed from $E_{v} \simeq E_{\mathrm{p}}+T_{n}+0.8 \mathrm{MeV}$, where $E_{\mathrm{p}}$ is the observed energy of the prompt signal and $T_{n}$ is the kinetic energy of neutron. With half-life about $207 \mu \mathrm{s}$, the neutron is captured by proton (carbon) and emits 2.2(4.9) $\mathrm{MeV} \gamma$-ray, which is called delayed event. Taking time-space correlation between these two events, we can observe electron anti-neutrino in almost background-free condition. 
ES channel cannot take advantage of the background suppression with delayed-coincidence and cannot provide the incident neutrino energy. However, this channel is sensitive to all flavors of neutrinos, though the cross section depends on the flavor of neutrino.

\section{Search for gravitational wave neutrinos}

Gravitational-wave experiment LIGO/Virgo collaboration founds a lot of burst events. They reported the event profiles in the second observing run (LIGO-O2) [5], the first half of the third observing run (LIGO-O3) [6], and the latest online alert on their public website [7]. From the gravitational wave source, thermal neutrino emission is theoretically predicted with the energy of a few tens $\mathrm{MeV}$ [8-10]. The follow-up searches for neutrinos have been performed by IceCube, Super-K, Borexino, and other various detectors [11-13]. KamLAND also reported the results for the first three gravitational wave events [14]. In this analysis, we searched $\bar{v}_{e}$ s associated with gravitational waves found in LIGO-O2 and -O3. A list of gravitational waves in LIGO-O2 and -O3 are taken from the published article [5] and their online gravitational wave candidate event database (GraceDB) [7], respectively. Taking into account some retraction of candidates in the GraceDB and the running status of the KamLAND detector at the timing of the gravitational wave event, we chose 60 gravitational waves summarized in Ref. [15].

We focused on $\bar{v}_{e}$ via the IBD channel in KamLAND with $0.9<E_{\mathrm{p}}<100 \mathrm{MeV}$ corresponding to $1.8<E_{v}<111 \mathrm{MeV}$. For the IBD selection, the energy of delayed event $\left(E_{\mathrm{d}}\right)$ should be $1.8<E_{\mathrm{d}}<2.6 \mathrm{MeV}$ or $4.4<E_{\mathrm{d}}<5.6 \mathrm{MeV}$. The spatial distance $(\Delta R)$ and time difference $(\Delta T)$ between the prompt and the delayed event are selected with $\Delta T<200 \mathrm{~cm}$ and $0.5<\Delta T<1000 \mu \mathrm{s}$, respectively. Besides, a likelihood-based signal selection was applied for rejecting radioactivityoriented background contamination. Muon and related spallation products are vetoed as standard KamLAND analysis [16]. Fiducial volume is restricted within a $6 \mathrm{~m}$ radius sphere from the center of the KamLAND, corresponding to $(5.98 \pm 0.13) \times 10^{31}$ of the number of target protons. For the KamLAND-Zen 400 and 800 phases, the delayed events in a $2.5 \mathrm{~m}$ radius sphere from the center or a $2.5 \mathrm{~m}$ cylindrical radius of the upper hemisphere are rejected to suppress the inner-balloon-related background contamination. In this analysis, a KamLAND dataset corresponding to LIGO-O3 is in the KamLAND-Zen 800 phase and excludes the inner-balloon volume. The neutrino detection efficiency for the LIGO-O2 and -O3 datasets are $\sim 93 \%$ and $\sim 77 \%$, respectively.

The neutrino event timing window was set as $\pm 500 \mathrm{~s}$ from each gravitational wave [17]. Using offtime from the gravitational waves, IBD-like events un-correlated with the gravitational waves are estimated to be $4.08 \times 10^{-3}$ and $4.27 \times 10^{-3}$ for gravitational wave events found in LIGO-O2 and $-\mathrm{O} 3$ periods, respectively. After the data interpretation, we found no $\bar{v}_{e}$ signals in that timing window and give an upper limit on the fluence with the Feldman-Cousins method [18] for each gravitational wave, assuming the monochromatic neutrino energy. Figure 1 shows the $\bar{v}_{e}$ fluence upper limits with $90 \%$ confidence level (C.L.) for each gravitational wave event found by LIGO-O2 and $-\mathrm{O} 3$. 


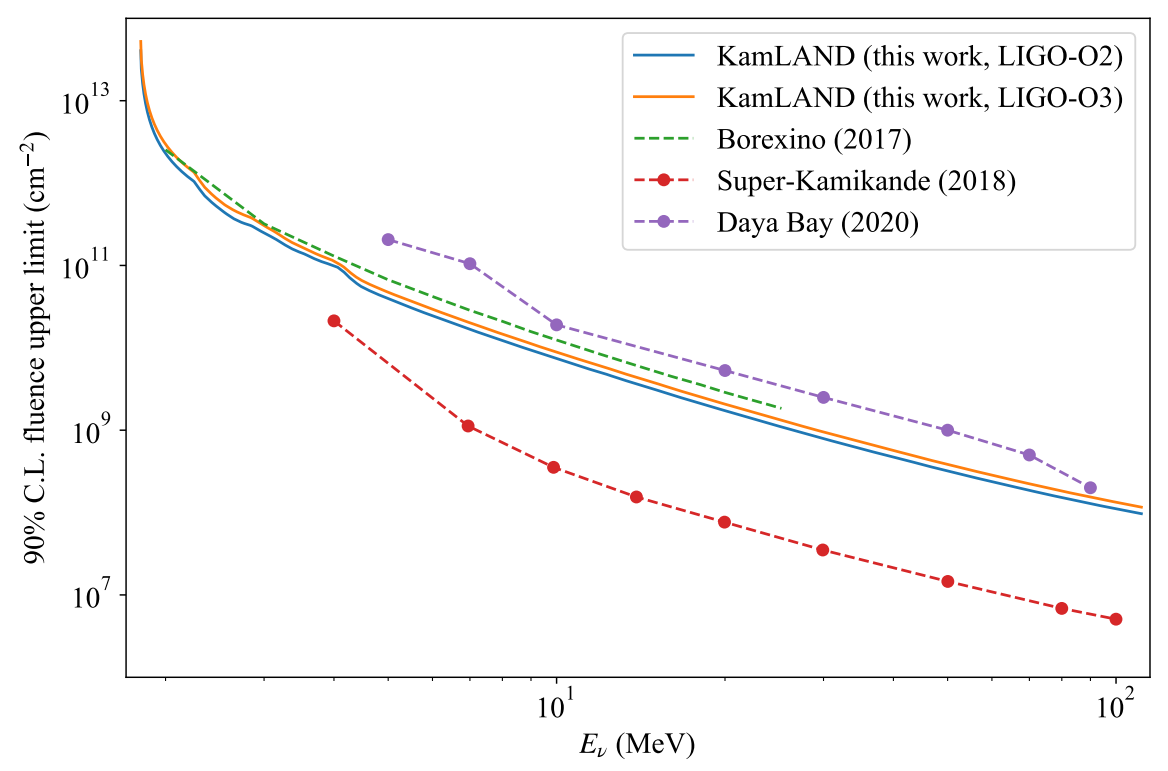

Figure 1: Upper limits on the $\bar{v}_{e}$ fluence with $90 \%$ C.L. Figure is reproduced from Ref. [15].

\section{Search for solar flare neutrino}

Solar flares are the largest explosive events in the solar system, which is described as reconnection of magnetic field and acceleration of charged particles. Observations of electromagnetic signals have contributed to the understanding of these burst phenomena [19]. Neutrinos are expected to be emitted from decays of charged pion as secondary particle of the initially-accelerated protons. Because solar flare neutrino spectrum depends on the profile of initially-accelerated charged particles, the observation of solar flare neutrino plays a key role in the understanding of particle acceleration mechanism in solar flare.

Some studies were developed to construct solar flare neutrino emission model [20-22]. An estimation from [22] predicts $398-770 \mathrm{~cm}^{-2}$ neutrino fluence per flare at Earth in $10-100 \mathrm{MeV}$, which corresponds to $O\left(10^{-9}\right)$ electron scatterings in KamLAND. For current neutrino detectors, it is hardly feasible to detect neutrino from a single flare. Thus, it is important to search for statistical excess using a number of flares.

The Homestake experiment reported an event excess possibly correlated to a large solar flare in 1991 [23], though subsequent studies by KAMIOKANDE II [24], LSD [25] and SNO [26] found no event excess related to solar flares. In 2019, Borexino performed a coincidence analysis of neutrino event candidates with solar flares selected from the Geostationary Operational Environmental Satellites (GOES) database and excluded the allowed parameter space for the solar flare neutrino fluence [27].

In this analysis, we searched neutrinos correlated with solar flares in assumption that the luminosity of solar flare neutrino is proportional to the soft X-ray luminosity as in Ref. [27]. From the GOES flare database, we chose 614 X- or M- class flares in our analysis period, from 2002 March to 2019 September, considering the KamLAND operation status. The time windows for flare neutrino search were determined using the derivative of the soft X-ray light curve based on 


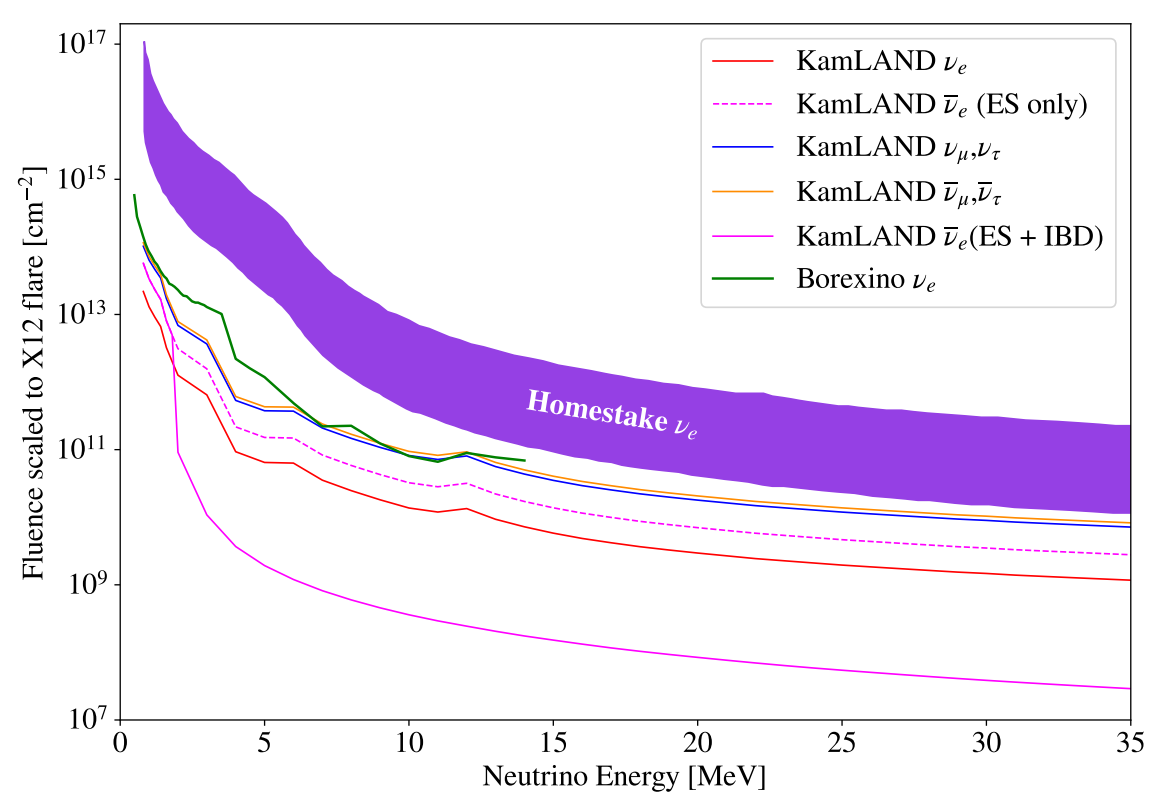

Figure 2: Fluence upper limits with $90 \%$ C.L. scaled to the Homestake flare intensity. Figure is reproduced from Ref. [29]

the method proposed in Ref. [28]. The average length of the 614 time windows is $1028 \mathrm{~s}$ and the cumulative flare intensity is $303 \times 10^{-4} \mathrm{~W} / \mathrm{m}^{2}$.

As for neutrino event selection, we used both IBD and ES in this analysis. The selection of IBD was the same as described in the previous section. For ES event selection, all single events remaining after muon-spallation-related event veto [3], ${ }^{238} \mathrm{U}$ decay series veto [16] and KamLANDZen volume cut(described above) are ES candidates. Assuming monochromatic solar flare neutrino spectrum, the analysis volume and the lower energy threshold to count the ES candidates were optimized for each assumed neutrino energy [29]. To take a coincidence analysis, the number of background events in each flare time window was estimated using offtime windows of each flare time window.

After a $\chi^{2}$ study with respect to the number of neutrino reactions (IBD/ES) by flare, we found no statistical excess related to solar flare and got $90 \%$ C.L. upper limits on the neutrino fluence from solar flares for each neutrino flavors and each neutrino energies. Figure 2 shows the fluence upper limits scaled to the Homestake flare intensity. This analysis provided a limit on unsearched parameter space for solar flare neutrino fluence.

\section{Summary}

KamLAND is a large-volume (anti-)neutrino detector which is sensitive to MeV-scale neutrinos.

We found no $\bar{v}_{e}$ events in KamLAND associated with the gravitational waves detected by the second and third LIGO/Virgo observing runs. The upper limits on the $\bar{v}_{e}$ fluence with $90 \% \mathrm{CL}$ are given for neutrino energies in the energy range of $1.8-111 \mathrm{MeV}$. 
Another analysis on solar flare neutrino in KamLAND was done and no statistical excess related to solar flares were found. KamLAND set the strongest limits on fluences of solar flare neutrinos in energy range of $0.4-35 \mathrm{MeV}$.

\section{Acknowledgments}

The KamLAND experiment is supported by JSPS KAKENHI Grants 19H05803; the World Premier International Research Center Initiative (WPI Initiative), MEXT, Japan; Netherlands Organization for Scientific Research (NWO); and under the U.S. Department of Energy (DOE) Contract No. DE-AC02-05CH11231, the National Science Foundation (NSF) No. NSF-1806440, NSF2012964, as well as other DOE and NSF grants to individual institutions. The Kamioka Mining and Smelting Company has provided services for activities in the mine. We acknowledge the support of NII for SINET4. This work is partly supported by the Graduate Program on Physics for the Universe (GP-PU). A part of this study was carried out by using the computational resources of the Center for Integrated Data Science, Institute for Space-Earth Environmental Research, Nagoya University through the joint research program.

\section{References}

[1] KAMLAND collaboration, Refurbishment of KamLAND Outer Detector, PoS ICHEP2016 (2017) 1161.

[2] A. Suzuki, Antineutrino science in KamLAND, EPJC 74 (2014) 3094.

[3] KamLAND-Zen Collaboration collaboration, Search for majorana neutrinos near the inverted mass hierarchy region with kamland-zen, PRL 117 (2016) 082503.

[4] KamLAND-Zen collaboration, First results of KamLAND-Zen 800, Journal of Physics: Conference Series 1468 (2020) 012142.

[5] Ligo Scientific Collaboration and Virgo Collaboration collaboration, Gwtc-1: A gravitational-wave transient catalog of compact binary mergers observed by ligo and virgo during the first and second observing runs, PRX 9 (2019) 031040.

[6] LigO Scientific Collaboration and Virgo Collaboration collaboration, Gwtc-2: Compact binary coalescences observed by ligo and virgo during the first half of the third observing run, $P R X 11$ (2021) 021053.

[7] LIGO Scientific Collaboration, "GraceDB (Accessed: 2020-07-04)." online. https://gracedb.ligo.org/superevents/public/O3/.

[8] F. Foucart, R. Haas, M.D. Duez, E. O'Connor, C.D. Ott, L. Roberts et al., Low mass binary neutron star mergers: Gravitational waves and neutrino emission, PRD 93 (2016) 044019.

[9] Y. Sekiguchi, K. Kiuchi, K. Kyutoku and M. Shibata, Gravitational waves and neutrino emission from the merger of binary neutron stars, PRL 107 (2011) 051102. 
[10] K. Kyutoku and K. Kashiyama, Detectability of thermal neutrinos from binary neutron-star mergers and implications for neutrino physics, PRD 97 (2018) 103001.

[11] SUPER-KAMIOKANDE collaboration, Search for neutrinos in coincidence with gravitational wave events from the ligo-virgo o3a observing run with the super-kamiokande detector, arXiv:2104.09196 [astro-ph.HE].

[12] M.G. Aartsen, M. Ackermann, J. Adams, J.A. Aguilar, M. Ahlers, M. Ahrens et al., Icecube search for neutrinos coincident with compact binary mergers from ligo-virgo's first gravitational-wave transient catalog, ApJ 898 (2020) L10.

[13] M. Agostini, K. Altenmüller, S. Appel, V. Atroshchenko, Z. Bagdasarian, D. Basilico et al., A search for low-energy neutrinos correlated with gravitational wave events gw 150914, gw 151226, and gw 170104 with the borexino detector, ApJ 850 (2017) 21.

[14] KAMLAND collaboration, A SEARCH FOR ELECTRON ANTINEUTRINOS ASSOCIATED WITH GRAVITATIONAL-WAVE EVENTS GW150914 AND GW151226 USING KAMLAND, ApJ 829 (2016) L34.

[15] KAMLAND collaboration, Search for low-energy electron antineutrinos in KamLAND associated with gravitational wave events, ApJ 909 (2021) 116.

[16] KAMLAND collaboration, SEARCH FOR EXTRATERRESTRIAL ANTINEUTRINO SOURCES WITH THE KamLAND DETECTOR, ApJ 745 (2012) 193.

[17] B. Baret, I. Bartos, B. Bouhou, A. Corsi, I.D. Palma, C. Donzaud et al., Bounding the time delay between high-energy neutrinos and gravitational-wave transients from gamma-ray bursts, APh 35 (2011) 1.

[18] G.J. Feldman and R.D. Cousins, Unified approach to the classical statistical analysis of small signals, PRD 57 (1998) 3873.

[19] A.O. Benz, Flare Observations Imprint / Terms of Use, LRSP 5 (2008) 1.

[20] G.E. Kocharov, G.A. Kovaltsov and I.G. Usoskin, Solar flare neutrinos, NCim C 14 (1991) 417.

[21] R. Takeishi, T. Terasawa and J. Kotoku, Numerical Studies of Neutrino Radiation in Solar Flares, in International Cosmic Ray Conference, vol. 33, p. 3656, Jan., 2013.

[22] ICECube collaboration, On the Study of Solar Flares with Neutrino Observatories, in 51st Rencontres de Moriond on EW Interactions and Unified Theories, pp. 573-576, 2016.

[23] R. Davis, A review of the homestake solar neutrino experiment, PrPNP 32 (1994) 13.

[24] K. Hirata, T. Kajita, T. Kifune, K. Kihara, M. Nakahata, K. Nakamura et al., Search for neutrino events in the kamiokande ii detector in correlation with the solar flare activity in 1989 march, ApJ 359 (1990) 574. 
[25] M. Aglietta et al., Large solar flares analysis of the events recorded by the Mont Blanc neutrino detector, ApJ 382 (1991) 344.

[26] B. Aharmim, S. Ahmed, A. Anthony, N. Barros, E. Beier, A. Bellerive et al., A search for astrophysical burst signals at the sudbury neutrino observatory, APh 55 (2014) 1-7.

[27] M. Agostini, K. Altenmüller, S. Appel, V. Atroshchenko, Z. Bagdasarian, D. Basilico et al., Search for low-energy neutrinos from astrophysical sources with borexino, APh 125 (2021) 102509.

[28] K. Okamoto, Y. Nakano, S. Masuda, Y. Itow, M. Miyake, T. Terasawa et al., Development of a method for determining the search window for solar flare neutrinos, SoPh 295 (2020) 133.

[29] KAMLAND collaboration, Search for Solar Flare Neutrinos with the KamLAND detector, arXiv:2105.02458 [astro-ph.SR]. 


\section{Full Authors List: KamLAND Collaboration}

N. Kawada ${ }^{1}$, S. Obara ${ }^{2}$, K. Ishidoshiro ${ }^{1}$, S. Abe ${ }^{1}$, S. Asami ${ }^{1}$, A. Gando ${ }^{1}$, Y. Gando ${ }^{1}$, T. Gima ${ }^{1}$, A. Goto ${ }^{1}$, T. Hachiya ${ }^{1}$, K. Hata ${ }^{1}$,

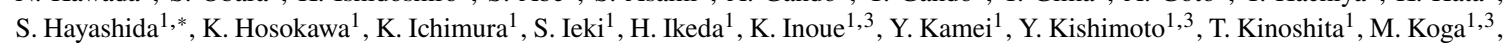
N. Maemura ${ }^{1}$, T. Mitsui ${ }^{1}$, H. Miyake ${ }^{1}$, K. Nakamura ${ }^{1}$, K. Nakamura ${ }^{1}$, R. Nakamura ${ }^{1}$, H. Ozaki ${ }^{1}{ }^{4}$, T. Sakai ${ }^{1}$, H. Sambonsugi ${ }^{1}$, I. Shimizu ${ }^{1}$, J. Shirai ${ }^{1}$, K. Shiraishi ${ }^{1}$, A. Suzuki ${ }^{1}$, Y. Suzuki ${ }^{1}$, A. Takeuchi ${ }^{1}$, K. Tamae ${ }^{1}$, K. Ueshima ${ }^{1, \dagger}$, Y. Wada ${ }^{1}$, H. Watanabe ${ }^{1}$,

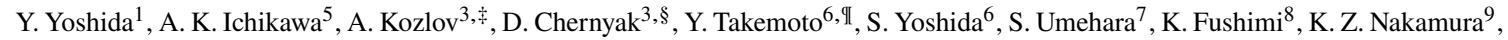
M. Yoshida ${ }^{9}$, B. E. Berger 10,3 , B. K. Fujikawa ${ }^{10,3}$, J. G. Learned ${ }^{11}$, J. Maricicic ${ }^{11}$, S. N. Axani ${ }^{12}$, L. A. Winslow ${ }^{12}$, Z. Fu ${ }^{12}$,

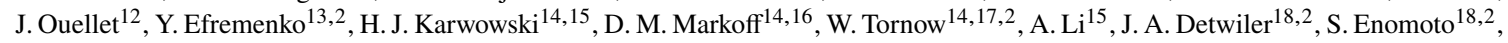
M. P. Decowski ${ }^{19,2}$, C. Grant ${ }^{20}$, T. O’Donnell ${ }^{21}$, S. Dell'Oro ${ }^{21}$

${ }^{1}$ Research Center for Neutrino Science, Tohoku University, Sendai 980-8578, Japan.

${ }^{2}$ Frontier Research Institute for Interdisciplinary Sciences, Tohoku University, Sendai, 980-8578, Japan.

${ }^{3}$ Institute for the Physics and Mathematics of the Universe, The University of Tokyo, Kashiwa 277-8568, Japan.

${ }^{4}$ Graduate Program on Physics for the Universe, Tohoku University, Sendai 980-8578, Japan.

${ }^{5}$ Department of Physics, Tohoku University, Sendai 980-8578, Japan.

${ }^{6}$ Graduate School of Science, Osaka University, Toyonaka, Osaka 560-0043, Japan.

${ }^{7}$ Research Center for Nuclear Physics (RCNP), Osaka University, Ibaraki, Osaka 567-0047, Japan.

${ }^{8}$ Graduate School of Advanced Technology and Science, Tokushima University, Tokushima, 770-8506, Japan.

${ }^{9}$ Department of Physics, Kyoto University, Kyoto 606-8502, Japan.

${ }^{10}$ Nuclear Science Division, Lawrence Berkeley National Laboratory, Berkeley, CA 94720, USA.

${ }^{11}$ Department of Physics and Astronomy, University of Hawaii at Manoa, Honolulu, HI 96822, USA.

${ }^{12}$ Massachusetts Institute of Technology, Cambridge, MA 02139, USA.

${ }^{13}$ Department of Physics and Astronomy, University of Tennessee, Knoxville, TN 37996, USA.

14 Triangle Universities Nuclear Laboratory, Durham, NC 27708, USA.

15 The University of North Carolina at Chapel Hill, Chapel Hill, NC 27599, USA.

${ }^{16}$ North Carolina Central University, Durham, NC 27701, USA.

${ }^{17}$ Physics Department at Duke University, Durham, NC 27705, USA.

${ }^{18}$ Center for Experimental Nuclear Physics and Astrophysics, University of Washington, Seattle, WA 98195, USA.

${ }^{19}$ Nikhef and the University of Amsterdam, Science Park, Amsterdam, The Netherlands.

${ }^{20}$ Boston University, Boston, MA 02215, USA.

${ }^{21}$ Center for Neutrino Physics, Virginia Polytechnic Institute and State University, Blacksburg, VA 24061, USA.

* Present address: Imperial College London, Department of Physics, Blackett Laboratory, London SW7 2AZ, UK.

$\dagger$ Present address: National Institutes for Quantum and Radiological Science and Technology (QST), Hyogo 679-5148, Japan.

$\ddagger$ Present address: National Research Nuclear University “MEPhI” (Moscow Engineering Physics Institute), Moscow, 115409, Russia. $\S$ Present address: Department of Physics and Astronomy, University of Alabama, Tuscaloosa, Alabama 35487, USA.

II Present address: Kamioka Observatory, Institute for Cosmic-Ray Research, The University of Tokyo, Hida, Gifu 506-1205, Japan. 